Des identités ouvertes et déployées : Trajectoire de vie d'un Arabe en ErE

Mohammed Taleb

\author{
(2) OpenEdition \\ Journals \\ Édition électronique \\ URL : http://journals.openedition.org/ere/501 \\ DOI : $10.4000 /$ ere.501 \\ ISSN : 2561-2271 \\ Éditeur \\ Centr'ERE
}

Référence électronique

Mohammed Taleb, "Des identités ouvertes et déployées : Trajectoire de vie d'un Arabe en ErE »,

Éducation relative à l'environnement [En ligne], Volume 12 | 2015, mis en ligne le 20 mai 2015, consulté le 21 février 2020. URL : http://journals.openedition.org/ere/501 ; DOI : 10.4000/ere.501 


\title{
Des identités ouvertes et déployées : Trajectoire de vie d'un Arabe en ErE
}

\author{
Mohammed Taleb
}

1 J'ai parfaitement conscience de la difficulté qu'il y a à parler de soi, de son itinérance, de sa trajectoire, fut-elle circonscrite à un domaine singulier, comme celui de l'Éducation relative à l'Environnement (ErE), et cela en inscrivant le «matériau biographique » dans la perspective générale d'une quête de sens. Dit autrement, je sais le risque que constitue la généralisation à partir d'un cas. C'est pourtant le pari que je souhaite faire à travers ces pages. Je n'ai nulle envie de pénétrer, en effet, dans la dialectique de l'engagement et de l'identité, qui est le grand thème de ce numéro, selon les règles d'une élucidation totalement abstraite et désincarnée. Cet article alternera donc descriptions et interprétations, avec le souci de ne pas raconter les événements d'un vécu singulier, mais de dire ce qui, en eux, peuvent porter des leçons pour tous. Mais j'aimerais, dès maintenant, mettre en évidence le point nodal de mon propos: plus la personne qui intervient dans le monde de l'éducation, de la formation, de la sensibilisation relatives à l'environnement est forte d'une multitude d'identités assumées, c'est-à-dire conscientisées, maîtrisées, et offertes (car le lien social porte irréductiblement une part de don de soi), plus elle a des chances d'inscrire sa démarche éducative et formatrice dans une perspective de globalité, mais aussi de résistance. La meilleure illustration, théorique et pratique, de cela est cette belle "Pédagogie de l'opprimé » du brésilien Paulo Freire (1921-1997), qui toute sa vie essaya de créer les conditions pour que, face au paradigme dominant de la modernité capitaliste, notamment dans sa logique de domination, d'exclusion et de silence imposé, les hommes, les femmes et les communautés qui sont dépossédés de leur savoir, de leur savoir-être et de leur dignité, accèdent à la parole et à la conscience de ce qu'ils sont (Freire, 1982). 


\section{Départ}

2 Je suis né en 1968 dans l'émigration algérienne en France; ma famille appartenait socialement à la classe ouvrière. Je me suis très tôt senti appelé à suivre deux filières, dont je pressentais qu'elles étaient profondément complémentaires, co-dépendantes. La première filière était celle d'un agir dans la sphère politique, en particulier dans les mouvements de la gauche arabe, dans les dynamiques de solidarité avec les luttes de libération nationale et anticoloniale, notamment celle du peuple palestinien. La seconde filière était liée à des préoccupations d'ordre spirituel et culturel, autour de mon arabité et de mon islamité. La politique et la spiritualité furent les deux grandes matrices où ma conscience se forgea. Dès mes années collégiennes et lycéennes, je me suis détourné du cadre éducatif formel, pour entrer dans l'action militante et dans la vie spirituelle. Ce sont ces deux voies qui me formèrent. Je peux donc dire que mon éducation fut largement une autoformation et une autodidactie. Dans les années 1990, j'ai suivi des cours à l'École des Langues et Civilisations de l'Orient Ancien (qui est l'une des composantes de l'Institut Catholique de Paris) et me suis inscrit à l'École Pratique des Hautes Études (dans sa section des sciences religieuses). Plus tard, au milieu des années 2000, j'ai entamé des études en Éducation relative à l'Environnement, au sein d'une formation proposée par l'Université du Québec à Montréal (UQAM). Le plus important ne résidait pas, pour moi, dans cette recherche du diplôme. Le défi était de mettre en cohérence, d'harmoniser, de synthétiser, de conjuguer ensemble les savoirs conceptuels et les processus existentiels, concrets-politiques et intimes-spirituels, qui étaient les miens. Dit autrement, il me fallait acquérir des clés d'analyses, des cadres interprétatifs, des structures de pensée afin de donner du sens et de la cohérence aux multiples engagements et aux nombreuses préoccupations qui étaient les miens. Mes questions étaient, par exemple : comment faire surgir de ma spiritualité islamique une perspective écologique? Comment articuler la critique sociale du capitalisme et cette «Pédagogie de l'imaginaire» qui me semblait être une voie d'excellence au sein de l'ErE ? Comment lier les enjeux de l'écoformation avec ceux de la conscientisation politique ? On le voit: ma quête était une quête des liaisons, des interactions et des correspondances.

3 Je voudrais partager ici les réflexions du philosophe Georges Gusdorf, qui a écrit des pages admirables sur les histoires de vie, l'écriture de soi et sur le romantisme aussi. Présentant, justement, la vision romantique (notamment allemande) de l'éducation, il écrivait ceci :

Le romantisme met en honneur le grand axe de la ligne de vie, autour duquel se regroupent les épisodes d'une existence, aventures et mésaventures à la faveur desquelles il est donné à un être humain de devenir ce qu'il est. Le vecteur éducatif se confond avec l'itinéraire de chacun vers lui-même, enquête expérimentale et conquête par chacun de sa propre authenticité. Cette difficile gestation ne se cantonne pas dans telle ou telle tranche d'âge ; elle embrasse le développement du sujet jusqu'à la maturité, et même au-delà, jusqu'à l'acquisition de cette sagesse propre aux vieillards. Dans ce projet global, l'instruction scolaire, si elle nécessaire, apparait insuffisante. La pédagogie romantique propose une méditation de la vie au cœur de la vie, appropriation par chacun de sa propre destinée. (Gusdorf, 1993, p. 382)

4 Quelques lignes plus loin, Georges Gusdorf souligne que l'autobiographie «s'attache aux significations plutôt qu'aux événements, à la personne et non au personnage ». C'est la raison pour laquelle, je souhaite illustrer maintenant cette relation entre le 
sens de mes engagements, en particulier au sein de l'ErE, et les multiples identités qui m'habitent. La conjugaison au pluriel de l'identitaire correspond à une réalité assez forte dans mon parcours. D'ailleurs, cette diversité est moins une juxtaposition qu'un entremêlement: identité politique (critique sociale $\mathrm{du}$ capitalisme et de l'impérialisme), identité culturelle (langue et imaginaire arabes en situation postcoloniale dans une métropole d'Occident). L'ensemble dessinant une perspective philosophique et spirituelle, entre philosophie néoplatonicienne, philosophie romantique et spiritualité éco-musulmane.

\section{Identité politique et engagement : pour une ErE subversive}

Si tu n'avais été d'abord un homme qui souffre et qui brûle, tu ne serais pas un homme qui pense.

Friedrich Hölderlin, Hypérion, 1797

De nombreux travaux publiés dans la revue Éducation relative à l'environnement: Regards - Recherches - Réflexions (notamment dans ses numéros 7, 8 et 9) ont mis en relief la nécessité d'une prise en compte de la dimension proprement politique de l'ErE, en lien avec la question des valeurs (éthique) et de la rationalité (pensée critique). Cette dimension est, semble- $\mathrm{t}$-il, la plus importante dans ma démarche, dans la mesure où elle est en lien immédiat avec la nécessité de procéder à une déconstruction radicale de la modernité capitaliste. Dans mon esprit, l'ErE poursuit essentiellement deux grands objectifs : réparation et transformation. Il s'agit de contribuer par l'éducation à la réparation des maux infligés par un système global qui malmène les relations socioécologiques, celles-ci constituant notre environnement. Mais si l'ErE se réduisait à cet objectif, elle ne manquerait de se voir manipuler et même de devenir un simple outil de verdissement superficiel, de réaménagement formel de nos sociétés, sans que soit remise en question la légitimité du paradigme dominant. En fait, c'est avec la seconde perspective, celle de la transformation, que le défi politique de l'ErE prend sa pleine puissance, car, avec lui, l'enjeu est celui de la contribution de l'ErE au dépassement d'une civilisation capitaliste occidentale, civilisation qui achève sa globalisation en suscitant, au Sud de la planète, l'émergence de pôles, des poches d'Occident (que ce soit en Inde, en Chine, dans les pays du Golfe arabique, en Amérique du Sud, etc.).

6 Dans mon itinérance, j'ai rencontré, j'ai éprouvé la difficulté d'articuler mon engagement politique (qui n'a jamais été un engagement partisan) avec une pratique d'éducateur ou de formateur. Si on considère que la critique sociale est au cœur de l'engagement politique, et que cette critique suppose nécessairement une distanciation avec les pouvoirs, il est facile de comprendre toute la difficulté de " politiser » l'ErE. En parlant de politisation de l'ErE, je veux parler de la participation de ses théoriciens et praticiens aux débats publics à propos des relations entre sociosphère et biosphère, débats qui engagent nos sociétés sur le moyen et le long terme. Lucie Sauvé le souligne en ces termes:

En milieu d'éducation formelle, l'exercice du raisonnement critique reste encore en marge du courant dominant de la transmission du savoir; mais surtout, la pédagogie critique (émancipatoire) a du mal à se déployer dans la culture institutionnelle ambiante. En milieu communautaire ou au sein des organisations citoyennes, la situation est tout aussi difficile : la rigueur critique doit s'installer à 
travers le bouillonnement des attitudes bien légitimes de colère, d'inquiétude ou d'impatience en situation de risque ou d'atteinte environnementale. (Sauvé, 2010-2011, p. 14)

7 Que signifie, dans mon esprit, "politiser » l'ErE ? Il ne s'agit certainement pas de lier l'ErE à un programme ou à un parti politique, mais de rappeler que la crise socioécologique planétaire, dont la résolution est, in fine, dans la ligne de visée de l'ErE, comprend une composante éminemment politique. C'est d'ailleurs cette dimension qui permet de résister à la thèse néolibérale et sociale-néolibérale qui veut que la résolution de cette crise passe nécessairement et même prioritairement par la décision technique. Or, nous savons tous que, même si elle a son importance, l'instance technique ne permettra jamais de régler des questions comme celles de nos modes de production, de consommation, de distribution, de la nature de propriété (sociale et/ou strictement privée), de la nature des relations internationales, etc. Ces questions sont clairement politiques et appellent un engagement, de nature politique, de tous ceux et de toutes celles qui aspirent à sortir de la crise socioécologique. L'ErE a toute sa place dans cette délibération, dans ce partage de la parole en vue du bien commun.

C'est à partir de ce constat que j'ai créé «ma » propre structure, à travers la voie associative, «Le singulier universel ». Son projet vise à promouvoir, au sein de l'ErE, de la mouvance écologiste et dans la société civile d'une façon plus globale, une compréhension de type transdisciplinaire de la crise environnementale (à partir de savoirs issus de sciences différentes, la physique, l'anthropologie, la psychologie, la sociologie, l'économie...), et une vigoureuse crique sociale de la «modernité capitaliste ». Depuis sa fondation en 1994, Le singulier universel a organisé des centaines de conférences, séminaires et d'interventions de toutes sortes dans des écoles, ou à l'université.

9 Je terminerai cette section en rappelant que dans ma trajectoire, l'articulation, au sein de l'ErE, entre engagement et identité politiques s'est faite sous le registre d'un marxisme débarrassé de ses tentations scientistes et économicistes, un marxisme plus près de certaines intuitions de Karl Marx lui-même qui avait su percevoir la relation intime entre capitalisme et crise socioécologique, et cela en plein 19e siècle, comme en témoigne ce passage du Capital (cité dans Löwy, 2003) :

La production capitaliste (...) détruit non seulement la santé physique des ouvriers urbains et la vie spirituelle des travailleurs ruraux, mais trouble encore la circulation matérielle (Stoffwechsel) entre l'homme et la terre, et la condition naturelle éternelle de la fertilité durable (dauernder) du sol, en rendant de plus en plus difficile la restitution au sol des ingrédients qui lui sont enlevés et usés sous forme d'aliments, de vêtements, etc. Mais en bouleversant les conditions dans lesquelles s'accomplit presque spontanément cette circulation, elle force de la rétablir d'une manière systématique, sous une forme adéquate au développement humain intégral et comme loi régulatrice de la production sociale. (...) En outre, chaque progrès de l'agriculture capitaliste est un progrès non seulement dans l'art d'exploiter le travailleur, mais encore dans l'art de dépouiller le sol; chaque progrès dans l'art d'accroître sa fertilité pour un temps est un progrès dans la ruine de ses sources durables de fertilité. Plus un pays, les États Unis du Nord de l'Amérique par exemple, se développe sur la base de la grande industrie, plus ce processus de destruction s'accomplit rapidement. La production capitaliste ne développe donc la technique et la combinaison du procès de production sociale qu'en sapant (untergräbt) en même temps les deux sources d'où jaillit toute richesse : la terre et le travailleur. 


\title{
Identité culturelle et engagement
}

\author{
Inscris ! \\ Je suis Arabe \\ Mes cheveux... couleur du charbon \\ Mes yeux... couleur de café Signes particuliers : \\ Sur la tête un keffieh avec son cordon bien serré \\ Et ma paume est dure comme une pierre \\ ... elle écorche celui qui la serre \\ La nourriture que je préfère c'est \\ l'huile d'olive et le thym. \\ Mahmoud Darwich, Identité, 1964
}

10 Si l'humanité existe comme "sujet ", elle ne se révèle pas seulement dans son unité, mais aussi dans sa diversité. Le pluralisme des trajectoires historiques, la diversité des aires de civilisation, la multiplicité des langues et des imaginaires, loin d'être antithétiques à l'universel, représentent la façon qu'à l'humanité de déployer les potentialités créatrices qu'elle porte en elle. C'est à travers le multiple que l'un se manifeste. Si le capitalisme heurte de plein fouet les équilibres socioécologiques de la terre, il malmène aussi la diversité historique qui est en grande partie une diversité d'ordre culturel. L'historien et sociologue Immanuel Wallerstein (1987) a très bien montré en quoi l'« universalisme » fut (et c'est encore largement le cas, par exemple avec le Développement durable !) l'une des composantes majeures du dispositif culturel et idéologique de la « modernité capitaliste».

L'universalisme représente toute une épistémologie. C'est un système de croyances relatives à l'objet du savoir et aux méthodes de la connaissance. Ce système a pour fondement essentiel l'idée qu'il est possible de formuler des propositions générales permettant d'interpréter le monde de façon significative - qu'il s'agisse du monde physique ou du monde social -, que ces propositions ont une validité universelle et permanente, et que l'activité scientifique a précisément pour objet d'établir de semblables propositions générales, sous une forme excluant tout élément prétendu subjectif, c'est-à-dire historiquement déterminé (...) La croyance en l'universalisme a constitué la pierre angulaire de l'édifice idéologique sur lequel s'est appuyé le capitalisme historique (...) L'universalisme recelait un piège ; en effet, il ne s'est pas imposé comme une idéologie libre et spontanée, mais a été propagé par les détenteurs du pouvoir dans le système- monde capitaliste. L'universalisme était offert au monde comme un cadeau des puissants aux faibles. (Wallerstein, 1987, pp. 79-84)

11 C'est précisément contre, résolument contre, cet universalisme-là, qui est d'abord une occidentalisation, que je me suis engagé, au sein de l'ErE, dans une démarche de valorisation de la diversité culturelle. Cette démarche, j'en suis persuadé, est l'antidote à la théorie néoconservatrice du choc des civilisations et aux divers replis identitaires qui peuvent émerger ici ou là. Pour ma part, la valorisation de mon identité culturelle arabo-musulmane dans mon engagement de formateur se fait clairement dans cette optique, que le public auquel je m'adresse soit culturellement arabe ou européen, ou autre. C'est dans l'imaginaire de ma culture que je puise les ressorts affectifs de mon attachement à la Terre en tant qu'elle est notre habitat à tous. Mais, cela ne signifie nullement que je n'éprouve pas également une passion pour les autres représentations culturelles de notre cosmos, de notre Nature vivante. Depuis plusieurs années, par exemple, j'essaie de souligner l'importance de la tradition indienne pour l'essor d'un monde multipolaire, y compris du point de vue la pensée écologique. Rabindranath Tagore (1861-1941) est une figure qui comptera certainement dans la recomposition 
symphonique, anticapitaliste, anti-impérialiste et écologique à laquelle j'appelle de mes vœux (Taleb 2012, 2011a, 2011b).

Loin de signifier un retour au passé ou un repli identitaire, la réhabilitation/ valorisation des patrimoines culturels de l'humanité, essentiellement pour les nations dominées, représente l'une des conditions les plus importantes du processus de constitution l'universel. C'est parce qu'il reposera sur cette diversité que l'universel évitera les impasses (parfois meurtrières, comme à l'époque coloniale) de l'universalisme capitaliste occidentalo-centré. Espaces de justice sociale, d'égalité, de démocratie, de liberté, d'écologie globale, de fraternité, de fécondité sociale et de vie spirituelle, les espaces culturels peuvent être ces territoires, ces lieux où les sujets locaux, régionaux et nationaux et continentaux échangent entre eux, en vue, non pas d'une accumulation du capital ou d'une exploitation de l'humain et du saccage de la Nature, mais, à l'inverse, pour faire tenir ensemble le singulier et l'universel. L'Éducation relative à l'Environnement, forte de plus de quarante ans d'expériences dans le monde entier, peut apporter une contribution, éducative, pédagogique, éthique et politique, à ce projet grandiose, celui de l'unus mundus des Alchimistes, celui de l'anima mundi des Anciens: les lieux que nous habitons sont habités. Mon père me disait : Mohammed, même lorsque tu entres dans une maison où il n'y a personne, tu dois quand même dire "as-salem alikoum ", à cause de la présence des anges, des djinns.

13 Célébrer la présence de l'esprit au cœur de la matière, du vivant au cœur de notre univers, de l'Âme au cœur de notre monde, de l'humain au cœur de la Nature, sont quelques-uns des enseignements, politiques et culturels, que je tire de mes engagements. Ils sont ce que je suis, dans une identité qui se conjugue au pluriel.

\section{BIBLIOGRAPHIE}

Darwich, M. (1989). Poèmes palestiniens. Chronique de la tristesse ordinaire. Paris : Le Cerf. Delory-Momberger, C. (2001). Bildung et écologie humaine : de la philosophie de la nature à la pédagogie de l'environnement. Éducation permanente, 148, 445-455. Freire, P (1982). La Pédagogie de l'opprimé. Paris : La Découverte/Maspéro. Gusdorf, G. (1993). Romantisme I. Paris : Payot.

Hölderlin, F. (1967). đeuvres. Paris : Gallimard/Bibliothèque de la Pléiade.

Löwy, M. (2003). Progrès destructif : Marx, Engels et l'écologie. Consulté le 9 novembre 2011 sur le site www.europe-solidaire.org/spip.php ?article370

Pineau, G., Bachelart, D., Cottereau, D. et Moneyron, A. (dir.) (2005). Habiter la Terre : Écoformation terrestre pour une conscience planétaire. Paris : l'Harmattan.

Sauvé, L. (2010-2011). La dimension politique de l'éducation relative à l'environnement - Un certain vertige. Éducation relative à l'environnement : Regards - Recherches - Réflexions, 9,7-21. 
Taleb, M. (2012). Ecopsychologie, science et spiritualité. Variations transdisciplinaire et transculturelle entre Inde, Islam et Occident. Rencontre avec l'Inde, Conseil Indien pour les relations Culturelles, tome 41, 1, 92-111.

Taleb, M. (2011a). Romain Rolland et Rabindranath Tagore. Une exploration goethéenne sur les chemins de l'Ame du monde Les Cahiers de Brèves, 28, décembre, pp. 4-12.

Taleb, M. (2011b). Tagore, l'Europe et l'Universel. Pour une approche goethéenne du dialogue des cultures. Rencontre avec l'Inde, Conseil Indien pour les relations Culturelles, tome 40, 1, 39-63.

Taleb, M. (2010-2011). A l'époque de la mondialisation capitaliste, trois défis pour l'éducation relative à l'environnement : l'écopsychologie, l'écodéveloppement et l'écosocialisme. Education relative à l'environnement. Regards - Recherches - Réflexions, 9, 25-44.

Wallerstein, I. (1987). Le capitalisme historique. Paris : La Découverte.

\section{INDEX}

Keywords : environmental education, ecopsychology, life history, identity, cultural identity, Arabic

Mots-clés : éducation relative à l'environnement, écopsychologie, histoire de vie, identité, identité culturelle, Arabe

\section{AUTEUR}

\section{MOHAMMED TALEB}

Philosophe algérien, enseigne l'écopsychologie et est formateur en éducation relative à l'environnement. Il s'est formé dans cette dernière discipline à l'Université du Québec à Montréal. Il préside l'association de philosophie « Le singulier universel ». Depuis de nombreuses années, il travaille sur les interactions entre écologie, spiritualité, critique sociale, dialogue interculturel et science. 